\title{
Leptospirosis bovina como causa de enfermedad reproductiva
}

\section{Bovine leptospirosis as a cause of reproductive failure}

\author{
Romero Becerra Liseth Rocio ${ }^{1}$ y Veloza Luis Carlos ${ }^{2}$ \\ ${ }^{1} \mathrm{MVZ}$. Universidad de los Llanos y ${ }^{2} \mathrm{MV}$. Esp Docente Universidad de los Llanos \\ lirobe 2408@hotmail.com
}

Recibido 27 de Junio 2014, Aceptado 03 de Octubre 2014

\section{RESUMEN}

La leptospirosis es una enfermedad infecciosa de carácter zoonótico y de distribución mundial, es caudada por espiroquetas del género Leptospira, especies: L. interrogans y $L$. biflexa (según clasificación fenotípica) o $L$. interrogans, L. biflexa y L. kirschneri (según clasificación genotípica). Los factores de virulencia asociados a Leptospiras patógenas son endotoxinas, hemolisinas esfingomielinasa, fosfolipasa y proteínas superficiales de adherencia. En los bovinos se ha encontrado Leptospira borgpetersenii Serovar hardjo, tipo hardjo bovis como un serovar adaptado, y Leptospira interrogans Serovar hardjo, tipo hardjo prajitmo de la especie interrogans, $L$. icterohaemorrhagiae, $L$. canicola y $L$. grippotyphosa como un serovares no adaptados. A nivel mundial Leptospira hardjo no es el único agente patógeno asociado a las fallas reproductivas y tampoco es la única serovariedad de Leptospira encontrada en bovinos. La inmunidad presentada frente a la leptospirosis es de tipo humoral, con un periodo de incubación de 4 a 10 días, se disemina en hígado, riñones, pulmones, tracto reproductor (placenta) y líquido cefalorraquídeo, produciendo daño al endotelio de los vasos sanguíneos, isquemia localizada en los órganos, necrosis tubular renal, daño hepatocelular y pulmonar, meningitis, miositis y placentitis. Las sustancias toxicas causan lisis de los eritrocitos y atraviesan la barrera placentaria produciendo la muerte fetal por anoxia terminando en aborto 1 o 2 días después.

Palabras clave: Aborto, leptospirosis, L. hardjo, L. prajitmo, placentitis. 


\begin{abstract}
Leptospirosis is a zoonotic infectious disease with global distribution, this disease is caudate spirochetal genus Leptospira species: L. interrogans and L. biflexa (as phenotypic classification) or L. interrogans, L. biflexa and L. kirschneri (based genotypic classification). The virulence factors associated with pathogenic Leptospira are endotoxins, hemolysins sphingomyelinase, phospholipase and surface adhesion proteins. In cattle found Leptospira borgpetersenii Serovar hardjo type hardjo bovis as an adapted serovar, and Leptospira interrogans serovar hardjo type hardjo prajitmo the species interrogans, L. icterohaemorrhagiae, L. canicola and L. grippotyphosa as a non-adapted serovars. Globally Leptospira hardjo is not the only pathogen associated with reproductive failure and it is not the only serovar of Leptospira found in cattle. The immunity presented against Leptospirosis is of humoral type. The microorganism has an incubation period of 410 days, spreads on liver, kidneys, lungs, reproductive tract (placenta) and cerebrospinal fluid, causing damage to the endothelium of blood vessels, organs localized ischemia, renal tubular necrosis, hepatocellular and lung damage, meningitis, myositis and placentitis. Toxic substances cause lysis of erythrocytes and cross the placenta causing fetal death by anoxia ending in 1-2 days after abortion.
\end{abstract}

Keywords: Abortion, leptospirosis, L. hardjo, L. prajitmo, placentitis.

\title{
INTRODUCCIÓN
}

Las Leptospiras son espiroquetas causantes de la enfermedad conocida como leptospirosis, asociada a falla reproductiva en bovinos presentando síntomas como infertilidad, aparición de mortinatos, abortos en el último tercio de la gestación y nacimiento de terneros débiles, pertenecen a la familia Leptospiraceae, se conocen alrededor de 220 serovares, la gran importancia de estos radica en que pueden estar presentes en muchas especies de mamíferos silvestres y domésticos. El diagnóstico rutinario de leptospirosis se hace mediante 
pruebas serológicas, la prueba oficial recomendada por la Organización Mundial de la Salud (OMS) es la prueba de microaglutinación (MAT).

Esta enfermedad es considerada prevalente y en ocasiones poco o mal diagnosticada, de impacto y estudio mundial, además de disminuir ostensiblemente los sistemas de producción, por lo cual es importante realizar una revisión y recopilación de literatura actualizada sobre las características etiológicas, epidemiológicas y fisiopatológicas causantes de problemas reproductivos en bovinos, con el fin de generar una base conceptual que ayude a los profesionales del sector pecuario involucrados en el manejo sanitario y productivo, y de esta manera obtener una producción eficiente y rentable, respetando las normas sanitarias y productivas nacionales, brindando productos alimenticios inocuos que protejan la salud humana.

La Leptospirosis es una enfermedad infecciosa aguda de carácter zoonótico, descrita por primera vez por Weil en 1886 (Pedraza et al., 2012), caracterizada por un amplio espectro de signos clínicos variando desde la infección inaparente a la enfermedad hasta el desarrollo mortal (Rodríguez, 2000), actualmente se distribuye en todo el mundo, presentándose principalmente en zonas tropicales con altos índices de precipitación (Rivera et al., 2012). En 1962 la subcomisión de Taxonomía de las Leptospiras de la Organización Mundial de la Salud acordó dividir a estas bacterias en dos especies: interrogans y biflexa, basándose en su comportamiento bioquímico, en la capacidad de infectar animales, resistencia a la acción de los iones de cobre bivalentes, en sus características biológicas y en las exigencias de cultivo (Araujo et al., 2010; Alonso et al., 2001; Zunino y Pizzarro, 2007; Pedraza et al., 2012), pero dicha clasificación taxonómica está siendo reemplazada por la clasificación genómica, la cual se realiza en base al estudio de hibridación del ADN bacteriano e identifica 20 genomoespecies (Levett, 2001; Alonso et al., 2001; Rivera et al., 2012). 


\section{DESCRIPCIÓN DEL AGENTE ETIOLÓGICO}

El termino Leptospira proviene del griego "Lepto" que significa fino y "spira" que significa espiral (Jiménez, 2006), son bacterias helicoidales, aerobias obligadas con la siguiente clasificación taxonómica: División Procariotes, clase Schizomicetes, orden Spirochaetales, familia Leptospiraceae, género Leptospira, especies: L. interrogans, L. biflexa (Laguna, 2000), miden de 20 a 30 um de largo por 0.2 a 0.3 um de ancho, son oxidasas positivas, no crecen en medios ordinarios de cultivo, pero sí lo hacen en medios suplementados con suero de conejo o en medio Tween 80-Albumina a un pH de 7.2-7.4 (Rodríguez, 2000), también crecen fácilmente en medios con Vitamina $B_{1}$ y $B_{12}$, ácidos grasos de cadena larga y sales de amonio, donde los ácidos grasos son utilizados como única fuente de carbono y metabolizados por $\beta$-oxidación; su temperatura optima de crecimiento es de 28 a $30^{\circ} \mathrm{C}$ (Adler y de la Peña, 2010). No sobreviven en el agua salada, al contrario de los largos períodos que pueden permanecer en el agua dulce principalmente si se encuentra almacenada (hasta 180 días); tampoco sobreviven en la leche salvo que ésta esté diluida en agua a la razón de 1:20 o más (Araujo et al., 2010). Son sensibles a la desecación, a la exposición directa, a los rayos solares, a pH menores de 5.8 o mayores de 8 y también se ven afectadas por las temperaturas extremas (Rodríguez, 2000). Se pueden almacenar por largos plazos en nitrógeno líquido, lo cual da buenos resultados y es considerado el medio perfecto de almacenamiento para mantener la virulencia (Adler y De la Peña, 2010).

Las Leptospiras son microorganismos que están constituidos por un cuerpo citoplasmático, un axostilo que se dispone en forma espiral y una membrana envolvente que recubre ambas estructuras. El axostilo consiste en dos filamentos axiales que se insertan en la extremidad del cuerpo citoplasmático, por medio de botones terminales, éste organelo es el encargado de la motilidad de la Leptospira. Al microscopio de campo oscuro puede observarse que una de las extremidades termina en gancho (Araujo et al, 2010) (Figura 1). Se ha descrito que este gancho es aerobio, diferenciado de otras espiroquetas patógenas y 
permite cultivarlas en medios artificiales (Acosta et al., 1994). Tienen dos flagelos periplásmicos con inserciones polares que son localizados en el espacio periplásmico y son responsables de la motilidad, estos contienen las proteínas FlaA y FlaB que constituyen la envoltura y el núcleo flagelar respectivamente (Adler y De la Peña, 2010). Puede sobrevivir por semanas o hasta 6 meses, dependiendo de las condiciones ambientales (Rodning, 2012).

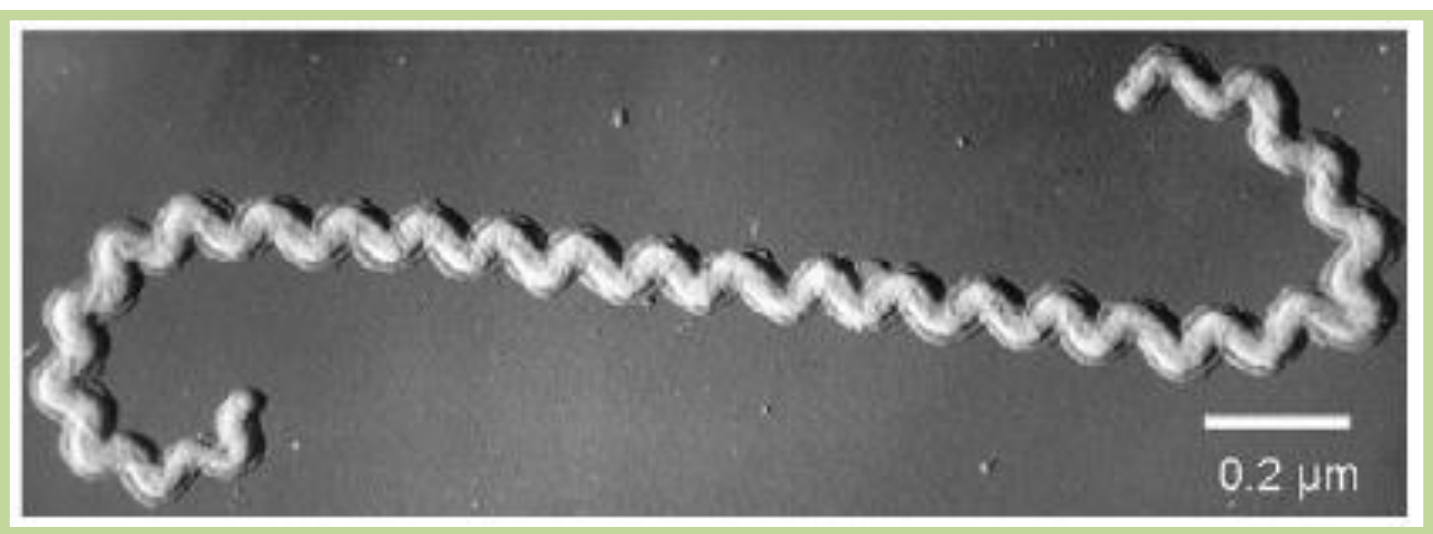

Figura 1. Microfotografía de Leptospira spp. Fuente: Adler y De la Peña, (2010)

Las Leptospiras tienen una estructura de doble membrana en la cual la membrana citoplasmática y los peptidoglicanos de la pared celular están estrechamente asociados y son superpuestos por una membrana externa, que contiene Lipopolisacaridos (LPS), proteínas estructurales y funcionales. Una gran proporción de estas proteínas son LPS con relativa abundancia en la superficie celular: LipL32, LipL21, LipL41; también contiene proteínas integrales de membrana tales como la porina OMP L1 y el sistema de secreción tipo 2 (T2SS), secretina GspD, de los cuales se ha demostrado que son antigénicos (Adler et al., 2011) (Figura 2). Las LipL21 y LipL41 están ubicadas en la superficie de la membrana exterior, y la LipL36 en el interior de la misma membrana. Las OMPL1 y LipL41 exhiben un sinergismo inmunosupresor según lo indican estudios realizados en hámster (Dey et al., 2004, Haake et al., 2004; Jiménez, 2006).

Se ha descrito que las OMPs en general que están expuestas en la superficie celular y en condiciones de interactuar con el medio ambiente contribuyendo a la patogénesis mediante la interacción con el hospedero, aunque se cree que la 
OMP L1 participa en la patogénesis sus funciones todavía no se han determinado (Cullen et al., 2004), así como la secuencia de la diversidad genómica y reactividad inmune cruzada entre diferentes serovares de Leptospiras patógenas sigue siendo desconocida (Dong et al., 2008).

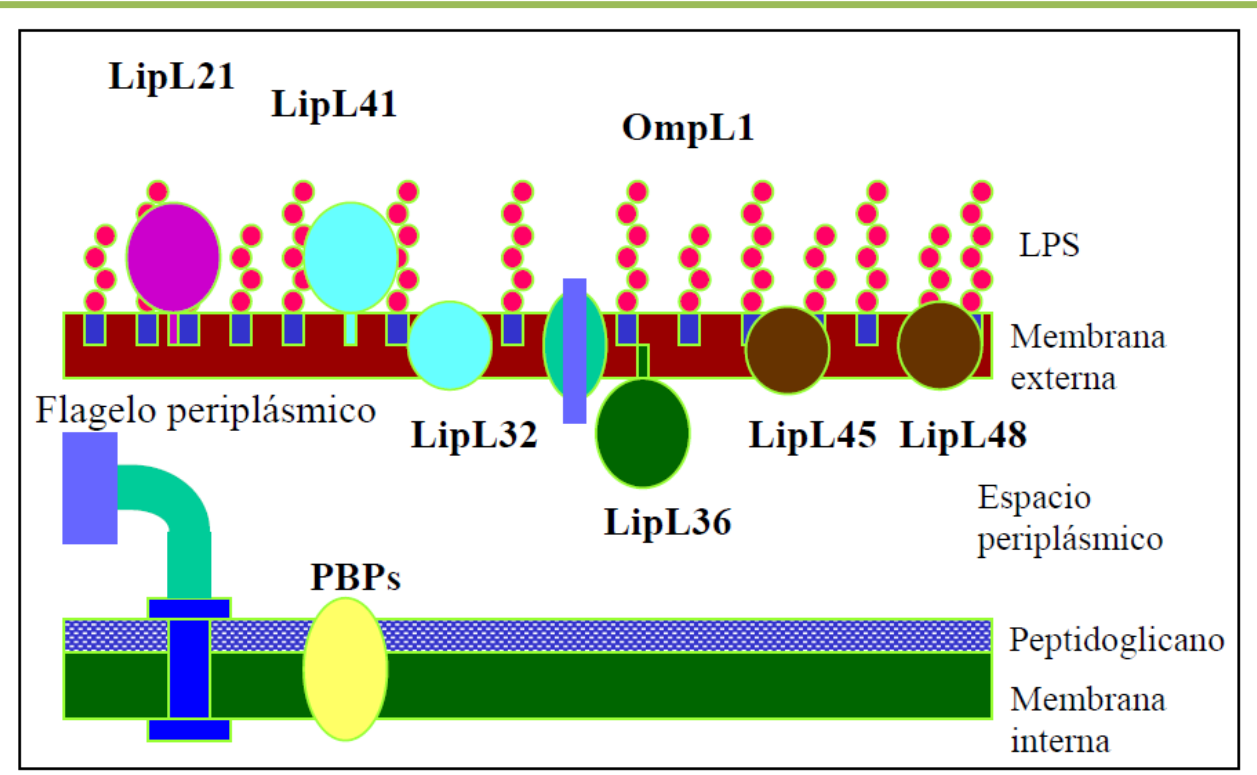

Figura 2. Estructura de la membrana celular Leptospira. Fuente: Jiménez, (2006)

En cuanto a la composición genómica de las Leptospiras se sabe que su cromosoma se caracteriza por un contenido de Guanina + Citocina de 35-41\% en moles, dependiendo de la especie, con un tamaño de genoma de 3.9-4.6 Mb. Actualmente hay seis secuencias del genoma publicados: dos serovares de $L$. interrogans (laik y copenhageni), dos cepas de L. borgpetersenii serovar hardjo y dos cepas de $L$. biflexa serovar patoc. Los genomas $L$ interrogans y $L$. borgpetersenii tienen dos cromosomas circulares y ambos se caracterizan por un alto grado de plasticidad, con evidencia de reordenación genómica a gran escala (Tabla 1). La diferencia más notable entre $L$. interrogans y $L$. borgpetersenii es que la primera tiene más pseudogenes que la segunda y sus secuencias de inserción, L. borgpetersenii es aproximadamente $700 \mathrm{~kb}$ más pequeño y tiene una densidad menor de codificación en comparación con L. interrogans. Significativamente, la pérdida de la función de genes no es aleatoria, pero está centrada en el deterioro de la detección del medio ambiente y el transporte y utilización de metabolitos. 
Estas características distinguen L. borgpetersenii de L. interrogans, una especie de decadencia genética mínima, y que sobrevive a paso prolongado en ambientes acuáticos que encuentran un hospedero mamífero. Por otro lado con $L$. borgpetersenii se observó que tiene un proceso de evolución hacia la dependencia de un ciclo de transmisión estricto de hospedero a hospedero (Adler y De la Peña, 2010).

Tabla 1. Características esenciales de los genomas de Leptospiras patógenas y saprófitas

\begin{tabular}{cccc}
\hline Característica & L. borgpetersenii & L. interrogans & L. biflexa \\
\hline Tamaño $(\mathrm{kb})$ & 3931 & 4627 & 3956 \\
Número de genes & 2844 & 3379 & 3590 \\
Codificación de densidad (\%) & 80 & 75 & 92 \\
Número de seudogenes & 368 & 41 & 33 \\
Número de transposasas & 246 & 26 & 9 \\
\hline
\end{tabular}

Fuente: Adler y de la Peña, (2010)

\section{ESPECIES PATÓGENAS Y NO PATÓGENAS}

Las Leptospiras patógenas se han incluido en la especie L. interrogans (250 serovares y 23 serogrupos), mientras que los serovares de vida libre no patógenos están incluidas en la especie L. biflexa (60 serovares) (Rodríguez, 2011). Las especies de Leptospiras consideradas patógenas son: L. borgpetersenii, L. indai, L. interrogans, L. kirschneri. L. noguchii, L. santarosai, L. weilii (Rodríguez, 2000), L. alexanderi, L. alstonii (genomospecies 1), L. fainei, L. licerasiae, L. santarosai, L. terpstrae y L. wolffii (Adler y De la Peña, 2010); y las consideradas no patógenas son: $L$. biflexa, $L$. meyeri $L$. wolbachii, $L$. yanagawae, L. kmetyi, L. vanthielii (Rodríguez, 2000). Se han descrito más de 220 serovares, (en la Tabla 2 se presenta un resumen de los serovares y serogrupos más representativos de L. interrogans), así como se ha determinado que la infección se produce por los serovares endémicos de una región o país y su presencia está ligada a los factores medioambientales y ecológicos (Alonso et al., 2001). 
Tabla 2. Resumen de los serogrupos y serovares más representativos de la especie L. interrogans.

\begin{tabular}{cl}
\hline Serogrupo & \multicolumn{1}{c}{ Serovar más Representativo } \\
\hline Sejroe & hardjo, saxkoebing, sejroe, wolffi \\
Autumnalis & Autumnalis \\
Ballum & ballum, castellonis \\
Bataviae & Bataviae \\
Canicola & Canicola \\
Cynopteri & Cynopteri \\
Grippotyphosa & Grippotyphosa \\
Hebdomadis & Hebdomadis \\
Icterohaemorrhagiae & copenhagheni, icterohaemorrhagiae \\
Javanica & javanica, poi \\
Louisiana & Louisiana \\
Mini & Swajizak \\
Pomona & Pomona \\
Pyrogenes & Pyrogenes \\
Sarmin & Sarmin \\
Australis & australis, Bratislava \\
Shermani & Shermani \\
Tarassovi & Tarassovi \\
\hline
\end{tabular}

Fuente: Alonso et al., (2001).

Actualmente el género se clasifica en 20 genomoespecies (clasificación genotípica) en base al estudio de hibridación del ácido desoxirribunucleico, estas a su vez, se clasifican en especies patógenas y saprófitas mediante la secuenciación del gen 16S rARN (Rivera et al., 2012), Esta clasificación ha sustituido la clasificación fenotípica, en el que un número de genomoespecies incluyen todos los serovares de los géneros $L$. interrogans, $L$. biflexa y $L$. kirschneri (Tabla 3) (Levett, 2001). La comparación de del genoma de estas tres especies reporta la identificación de 2052 genes en común para las mismas, lo cual es un hallazgo que indica un origen común para las Leptospiras saprofitas y patógenas a nivel del núcleo (Figura 2) (Adler y De la Peña, 2010). Las genomospecies de Leptospira no corresponden con las anteriores dos especies identificadas taxonómicamente (L. interrogans y L. biflexa), y de hecho, se reporta que los serovares patógenos y no patógenos pertenecen a la misma especie (Tabla 4). Así, ni el serogrupo ni el serovar predice con fiabilidad la especie de Leptospira (Tabla 5). En conclusión, la reclasificación taxonómica de las 
Leptospiras por genotipo correcta y proporciona una base sólida para futuras clasificaciones (Levett, 2001).

Tabla 3. Genomoespecies de Leptospira y distribución de serogrupos

\begin{tabular}{|c|c|}
\hline Especies & Serogrupos \\
\hline L. interrogans & $\begin{array}{l}\text { Icterohaemorrhagiae, Canicola, Pomona, Australis, Autumnalis, } \\
\text { Pyrogenes, Grippotyphosa, Djasiman, Hebdomadis, Sejroe, } \\
\text { Bataviae, Ranarum, Louisiana, Mini, Sarmin }\end{array}$ \\
\hline L. noguchii & $\begin{array}{l}\text { Panama, Autumnalis, Pyrogenes, Louisiana, Bataviae, Tarassovi, } \\
\text { Australis, Shermani, Djasiman, Pomona }\end{array}$ \\
\hline L. santarosai & $\begin{array}{l}\text { Shermani, Hebdomadis, Tarassovi, Pyrogenes, Autumnalis, } \\
\text { Bataviae, Mini, Grippotyphosa, Sejroe, Pomona, Javanica, Sarmin, } \\
\text { Cynopteri }\end{array}$ \\
\hline L. meyeri & Ranarum, Semaranga, Sejroe, Mini, Javanica \\
\hline L. wolbachii & Codice \\
\hline L. biflexa & Semaranga, Andamana \\
\hline L. fainei & Hurstbridge \\
\hline L. borgpetersenii & $\begin{array}{l}\text { Javanica, Ballum, Hebdomadis, Sejroe, Tarassovi, Mini, Celledoni, } \\
\text { Pyrogenes, Bataviae, Australis, Autumnalis }\end{array}$ \\
\hline L. kirschneri & $\begin{array}{l}\text { Grippotyphosa, Autumnalis, Cynopteri, Hebdomadis, Australis, } \\
\text { Pomona, Djasiman, Canicola, Icterohaemorrhagiae, Bataviae, }\end{array}$ \\
\hline L. weilii & $\begin{array}{l}\text { Celledoni, Icterohaemorrhagiae, Sarmin, Javanica, Mini, Tarassovi, } \\
\text { Hebdomadis, Pyrogenes, Manhao, Sejroe }\end{array}$ \\
\hline L. inadai & $\begin{array}{l}\text { Lyme, Shermani, Icterohaemorrhagiae, Tarassovi, Manhao, } \\
\text { Canicola, Panama, Javanica }\end{array}$ \\
\hline L. parva & Turneria \\
\hline L. alexanderi & Manhao, Hebdomadis, Javanica, Mini \\
\hline
\end{tabular}

Fuente: Levett, (2001).

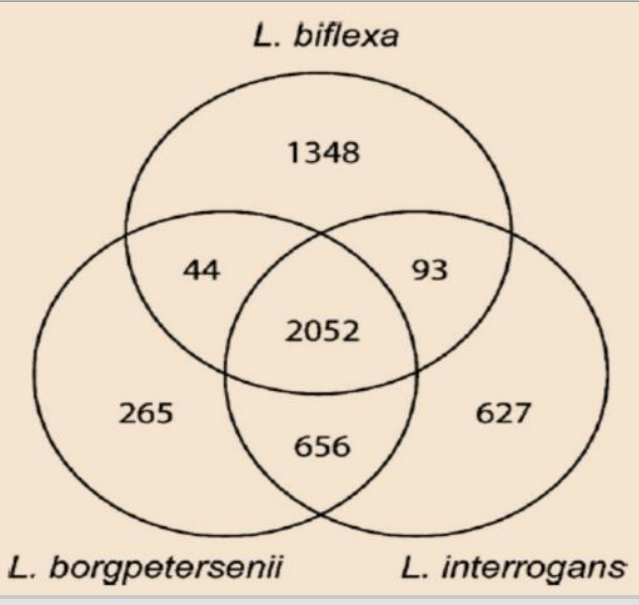

Figura 3. Genoma comparado de Leptospira spp. Número de genes individuales y en común entre las especies $L$. biflexa, $L$. borgpeternsenii y L. interrogans. Fuente: Adler y De la Peña, 2010 
Tabla 4. Genomoespecies asociadas con serogrupos

\begin{tabular}{|c|c|}
\hline Serogrupo & Genomoespecies \\
\hline Andamana & L. biflexa \\
\hline Australis & $\begin{array}{l}\text { L. interrogans, L. noguchii, L. borgpetersenii, L. } \\
\text { kirschneri }\end{array}$ \\
\hline Autumnalis & $\begin{array}{l}\text { L. interrogans, L. noguchii, L. santarosai, L. } \\
\text { borgpetersenii, L. kirschneri }\end{array}$ \\
\hline Ballum & L. borgpetersenii \\
\hline Bataviae & $\begin{array}{l}\text { L. interrogans, L. noguchii, L. santarosai, } L \text {. } \\
\text { borgpetersenii, L. Kirschneri }\end{array}$ \\
\hline Canicola & L. interrogans, L. inadai, L. kirschneri \\
\hline Celledoni & L. weilii, L. borgpetersenii \\
\hline Codice & L. wolbachii \\
\hline Cynopteri & L. santarosai, L. kirschneri \\
\hline Djasiman & L. interrogans, L. noguchii, L. kirschneri \\
\hline Grippotyphosa & L. interrogans, L. santarosai, L. Kirschneri \\
\hline Hebdomadis & $\begin{array}{l}\text { L. interrogans, L. weilii, L. santarosai, L. borgpetersenii, } \\
\text { L. kirschneri, L. alexanderi }\end{array}$ \\
\hline Hurstbridge & L. fainei \\
\hline Icterohaemorrhagiae & L. interrogans, L. weilii, L. inadai, L. Kirschneri \\
\hline Javanica & $\begin{array}{l}\text { L. weilii, L. santarosai, L. borgpetersenii, L. meyeri, L. } \\
\text { inadai, L. alexanderi }\end{array}$ \\
\hline Louisiana & L. interrogans, L. noguchii \\
\hline Lyme & L. inadai \\
\hline Manhao & L. weilii, L. inadai, L. alexanderi \\
\hline Mini & $\begin{array}{l}\text { L. interrogans, L. weilii, L. santarosai, L. borgpetersenii, } \\
\text { L. meyeri, L. alexanderi }\end{array}$ \\
\hline Panama & L. noguchii, L. inadai \\
\hline Pomona & L. interrogans, L. noguchii, L. santarosai , L. kirschneri \\
\hline Pyrogenes & $\begin{array}{l}\text { L. interrogans, L. noguchii, L. weilii, L. santarosai, L. } \\
\text { borgpetersenii }\end{array}$ \\
\hline Ranarum & L. interrogans, L. meyeri \\
\hline Sarmin & L. interrogans, L. weilii, L. santarosai \\
\hline Sejroe & $\begin{array}{l}\text { L. interrogans, L. weilii, L. santarosai, L. borgpetersenii, } \\
\text { L. Meyeri }\end{array}$ \\
\hline Semaranga & L. meyeri, L. biflexa \\
\hline Shermani & L. noguchii, L. santarosai, L. inadai \\
\hline Tarassovi & $\begin{array}{l}\text { L. noguchii, L. weilli, L. santarosai, L. borgpetersenii, L. } \\
\text { Inadai }\end{array}$ \\
\hline
\end{tabular}

Fuente: Levett, (2001). 
Tabla 5. Serovares de Leptospira que se encuentran en diferentes especies

\begin{tabular}{cl}
\hline Serovar & \multicolumn{1}{c}{ Especies } \\
\hline Bataviae & L. interrogans, L. santarosai \\
Bulgarica & L. interrogans, L. kirschneri \\
Grippotyphosa & L. kirschneri, L. interrogans \\
Hardjo & L. borgpetersenii, L. interrogans, L.meyeri \\
icterohaemorrhagiae & L. interrogans, L. inadai \\
Kremastos & L. interrogans, L. santarosai \\
Mwogolo & L. kirschneri, L. interrogans \\
Paidjan & L. kirschneri, L. interrogans \\
Pomona & L. interrogans, L. noguchii \\
Pyrogenes & L. interrogans, L. santarosai \\
Szwajizak & L. interrogans, L. santarosai \\
Valbuzzi & L. interrogans, L. kirschneri
\end{tabular}

Fuente: Levett, (2001).

\section{FACTORES DE VIRULENCIA}

Se refieren a las propiedades que permiten a un microorganismo establecerse en o dentro de un hospedero para mejorar su potencial de causar enfermedad, son toxinas bacterianas, proteínas de la superficie celular que median la adhesión bacteriana, carbohidratos de la superficie celular y proteínas que protegen la bacteria, y las enzimas hidrolíticas que pueden contribuir a la patogenicidad de la bacteria (Chen et al., 2012), es así que las Leptospiras patógenas tienen factores de virulencia como endotoxinas, hemolisinas, esfingomielinasa, fosfolipasa y proteínas superficiales de adherencia (Acosta et al., 1994). Las endotoxinas en varios serovares de Leptospiras (Levett, 2001), se definen como toxinas termoestables ubicadas en la membrana externa, no son excretadas por las bacterias sino que se liberan cuando las células son disgregadas, son menos tóxicas que las exotoxinas y no forman toxoides (IQB, 2010).

Las hemolisinas que se encuentran en un gran número de serovares de Leptospira ballum, hardjo, pomona, y tarassovi se han caracterizado como esfingomielinasas (Adler y de la Peña, 2010), donde las cepas virulentas exhiben quimiotaxis hacia la hemoglobina, en plasma se ha demostrado que previene la hemólisis (Levett, 2001). Una serie de genes que codifican esfingomielinasas son bien asociados al interior celular y zona extracelular; actualmente se ha identificado en diferentes especies de Leptospiras, al menos siete genes sphA 
similares se detectaron entre las especies patógenas de Leptospira, incluyendo un postulado SphH formador de poros hemolisina (Adler y de la Peña, 2010). La actividad de la fosfolipasa $C$ se ha informado en serovar canicola (Levett, 2001).

Las proteínas superficiales de adherencia que se encuentran en la estructura de doble membrana de las Leptospiras que constituyen los antígenos principales son similares estructuralmente e inmunológicamente a los lipopolisacaridos (LPS) de los organismos gram negativos. (Adler y de la Peña, 2010). Adicionalmente las Leptospiras cuentan con una lipoproteína L32 (LipL32), también conocida como Hap1, que constituye más del $50 \%$ de las sub-proteínas de la membrana externa, (lipoproteínas de superficie expuestas), son las proteínas más abundantes en la célula; se encuentran exclusivamente en Leptospiras patógenas, se expresan in vivo y son altamente inmunogénicas (Adler y de la Peña, 2010). La LipL32 la más importante en la membrana externa, siendo la defensora principal a la respuesta de anticuerpos humanos y animales (Cao et al., 2010), se ha demostrado que se une a varias proteínas de la matriz extracelular, incluyendo laminina, múltiples colágenos y fibronectina, plasminógeno y proteínas plasmáticas, lo cual indica que también puede ser necesaria para la supervivencia en el medio ambiente, aunque todas estas posibilidades siguen siendo tema de estudio (Murray, 2013).

\section{EPIDEMIOLOGÍA}

La leptospirosis es considerada una enfermedad reemergente, de distribución mundial, de comportamiento endémico y con brotes en varios continentes (Ochoa et al., 2000). Los reservorios de las Leptospirosis son atribuidos a una serie de animales salvajes y domésticos donde la serovariedad de la Leptospira infectante varía de acuerdo con el animal afectado; sin embargo los serotipos no son necesariamente específicos de una especie animal, porque pueden aparecer en: bovinos con el serovar predominante shermani y pomona, cerdos con bratislava y además de perros con shermani y canícola. Otros reservorios han sido mencionados como portadores: venados, ciervos, ardillas, zorros, mapaches, marsupiales y leones de mar. Inclusive fueron encontrados anticuerpos en crotálidos y se ha reportado que las variedades que infectan a los reptiles y 
anfibios al parecer no infectan al ser humano (Araujo et al., 2010). En conclusión, una o más especies de mamíferos silvestres y domésticos, actúan como hospedadores de cada serovar de Leptospiras patógenas, indicando que una especie animal es reservorio de varios serovares y diferentes especies animales son reservorio de un mismo serovar (Alonso et al., 2001). Diferentes estudios demuestran que se han presentado infecciones con $L$. interrogans en animales y en humanos, encontrándose los serovares $L$. icterohemorragiae, $L$. pomona, $L$. grippotyphosa, L. canicola, L. tarssovi (Maris y Rango, 2011), L. hardjo (Givens, 2008) y L. ballum (Pedraza et al., 2012) como las más prevalentes. Debido a esto la enfermedad no puede considerarse o tratarse como un problema individual sino como un problema del hato, o de un grupo de animales de un ecosistema. A medida que la enfermedad se hace endémica la severidad clínica disminuye debido a los mecanismos de resistencia o a la disminución de la patogenicidad del microorganismo, manifestándose como una patología reproductiva en los animales más susceptibles como forma crónica (Alfaro, 2004).

La mayoría de las serovariedades de Leptospira están asociadas a una especie animal determinada, por ejemplo: L. pomona y L. interrogans serovar hardjo (Rodning, 2012) asociadas a bovinos y cerdos, L. grippotyphosa en bovinos y corderos, $L$. ballum y $L$. icterohaemorrhagiae son asociadas a roedores y $L$. canicola en perros (Odriozola, 2001). Las diferentes cepas patógenas de Leptospiras afectan potencialmente a un gran número de especies animales, que actúan como hospedador de mantenimiento o accidentales, en función de la adaptación o preferencia del serovar involucrado (Alfaro, 2004) (Tabla 6). Un animal puede estar infectado por serovares mantenidos por su propia especie o serovares mantenidos por otras especies (Rodning, 2012). Se ha observado que algunos animales siendo hospederos naturales de un serovar en particular, generalmente no reflejan o muestran pocos efectos de la enfermedad frente a ese serovar, pero pueden llegar a desarrollar los síntomas de la enfermedad si son infectados con un serovar distinto, esto supone entonces, que la Leptospira induce inmunidad de tipo humoral que solo protege frente al serovar infectante, indicando 
que estos animales inmunes pueden actuar como fuente de infección (Rodríguez, 2011).

Tabla 6. Diferencias entre hospedador de mantenimiento y accidental

\begin{tabular}{|c|c|c|}
\hline Aspecto & $\begin{array}{l}\text { Hospedador de } \\
\text { Mantenimiento }\end{array}$ & Hospedador Accidental \\
\hline $\begin{array}{l}\text { Transmisión de } \\
\text { Leptospiras }\end{array}$ & Frecuente intraespecie & Esporádica intraespecie \\
\hline $\begin{array}{l}\text { Signos de enfermedad } \\
\text { aguda }\end{array}$ & Benignos (agalactia) & $\begin{array}{c}\text { Graves: complicaciones } \\
\text { hepáticas y renales }\end{array}$ \\
\hline $\begin{array}{l}\text { Signos de enfermedad } \\
\text { crónica }\end{array}$ & $\begin{array}{c}\text { Problemas reproductivos, } \\
\text { uveítis }\end{array}$ & Aparentemente ninguno \\
\hline $\begin{array}{l}\text { Duración de la } \\
\text { leptospiruria }\end{array}$ & $\begin{array}{l}\text { Prácticamente toda la } \\
\text { vida }\end{array}$ & Días a semanas \\
\hline $\begin{array}{l}\text { Porcentaje de población } \\
\text { seropositiva }\end{array}$ & $\begin{array}{l}\text { Alta, aumenta con la } \\
\text { edad de los animales }\end{array}$ & $\begin{array}{c}\text { Baja, No afectada con la } \\
\text { edad de los animales }\end{array}$ \\
\hline \multirow[t]{2}{*}{$\begin{array}{l}\text { Muestras para el } \\
\text { diagnóstico }\end{array}$} & Rebaño & Animal afectado \\
\hline & EJEMPLOS DE SEROVARES & \\
\hline Bovinos & hardjo, Pomona & grippotyphosa \\
\hline Porcinos & Bratislava & autumnalis \\
\hline $\begin{array}{c}\text { Perro (enfermedad de } \\
\text { Stuttgart) }\end{array}$ & Canícola & icterohaemorrhagiae \\
\hline Caballo & Bratislava & pomona \\
\hline Roedores & Icterohaemorrhagiae & pomona \\
\hline Ovinos y Caprinos & ballum, hardjo & Pomona \\
\hline $\begin{array}{l}\text { Hombre (enfermedad de } \\
\text { Weil) }\end{array}$ & Icterohaemorrhagiae & Variedad \\
\hline
\end{tabular}

Fuente: Alfaro, (2004).

Las Leptospiras patógenas ( $L$. interrogans) solo se multiplican dentro de los organismos animales por lo que los focos de Leptospirosis precisan de condiciones ambientales favorables para la supervivencia del agente causal en el medio exterior (Araujo et al., 2010). La transmisión de Leptospira es tanto horizontal como vertical en los animales y hospedadores de serovares adaptados, (Bermúdez, 2008). Se puede transmitir de forma directa por medio de contacto con orina, descargas uterinas, restos de placenta (después de un aborto), en forma venérea, por vía transplacentaria (infecciones congénitas) (Gasque, 2008), por la 
glándula mamaria (Pérez, 1985), o por forma indirecta, por medio del agua de bebida, pastos y/o alimentos. La Leptospira interrogans, serovariedad hardjo, se excreta por el aparato genital durante el aborto e incluso hasta 8 días después de que se produce el mismo (Gasque, 2008). Entran al hospedador a través de las membranas mucosas o abrasiones dérmicas y difusión hematógena (Murray, 2013). En bovinos la transmisión de serovares como hardjo es más frecuente de forma horizontal directa, mientras que la transmisión horizontal indirecta tiene un papel importante en infecciones accidentales, posterior a la exposición del animal a un ambiente contaminado con material infectado por Leptospira (Ellis, 1994). Una transmisión por contacto directo puede producirse por muchas maneras, siendo una de las más importantes el ingreso del agente patógeno por vía respiratoria o conjuntival, proveniente de núcleos goticulares formados por la dispersión de la orina de animales infectados (Alonso et al., 2001). La infección se puede dar debido a que muchos hospedadores de mantenimiento de un determinado serovar eliminan gran cantidad de microorganismos en su orina durante un periodo de tiempo prolongado, por lo que las gloticulas de orina tendrán una alta concentración de gérmenes y agentes patógenos. Igualmente, también se indica que este hecho, unido a la alta receptibilidad de los hospedadores de mantenimiento a la infección por un serovar adaptado, supone que esta forma de transmisión juega un papel importante (Alonso et al., 2001). Otra forma de transmisión es la indirecta, la cual tiene un papel más representativo en las infecciones accidentales; una forma de transmisión frecuente en el hombre y animales es el contacto de la piel erosionada o mucosas con agua o barro contaminados con orina infectada (Alonso et al., 2001) (Tabla 7).

\section{FALLA REPRODUCTIVA EN BOVINOS}

En bovinos la Leptospira borgpetersenii Serovar hardjo, tipo hardjo bovis fue caracterizada como un serovar adaptado y Leptospira interrogans Serovar hardjo, tipo hardjo prajitno de la especie interrogans como un serovar no adaptado, estos biotipos (serovar) son muy similares en la inmunogenicidad, no comportándose similarmente en la antigenicidad, resultando indistinguibles en la prueba de 
microaglutinación (MAT), a pesar que son molecularmente diferentes (Bermúdez, 2008).

Tabla 7. Hospedadores accidentales y de mantenimiento en animales y el hombre

\begin{tabular}{ccc}
\hline Leptospira spp. & $\begin{array}{c}\text { Hospedador de } \\
\text { mantenimiento }\end{array}$ & Hospedador accidental \\
\hline hardjo-bovis & Ganado & Ovejas, humanos \\
hardjo-prajitmo & Ganado & Ovejos, humanos \\
pomona & Cerdos & Ganado, cerdos \\
canicola & Perros & Ganado, cerdos \\
grippotyphosa & Mapaches & Ganado, cerdos \\
icterohemorragiae & Ratas & Ganado, cerdos \\
\hline
\end{tabular}

Fuente: Ramírez, (2008).

Otros serovares de Leptospira no adaptados a los bovinos son icterohaemorrhagiae, canicola y grippotyphosa, los cuales causan signos clínicos similares a los presentados por L. hardjo (Rodning, 2012). La Leptospira borgpetersenii serovar Hardjo Tipo hardjo-bovis se reporta con más frecuencia en América y Australia, mientras que Leptospira interrogans serovar Hadjo Tipo hardjo-prajitmo es encontrada más frecuentemente en el Reino Unido y Europa; la infección por L. borgpetersenii serovar Hardjo Tipo hardjo bovis es la causa más común de Leptospirosis bovina en ganado de leche y carne en algunos países del continente americano (Bermúdez, 2008), y se encuentra altamente correlacionada con pérdidas embrionarias en Brasil, Perú y Ecuador (Rodríguez, 2003), Suiza, Australia, México, EE.UU e Irlanda (Ryan et al., 2012).

Cangahuamin en el 2011 realizó un estudio en 138 hembras bovinas con el fin de determinar las principales causas de problemas reproductivos en el estado de Sangolquí, Ecuador; en este estudio se realizaron las pruebas específicas para las diferentes enfermedades así; Brucelosis (Rosa de bengala), DBV (Elisa competitiva); IBR (Elisa competitiva), Leptospirosis (MAT) y Neospora (Elisa competitiva); los parámetros reproductivos evaluados evidenciaron que el $38 \%$ de las vacas tenían más de 90 días abiertos y el $8 \%$ había presentado aborto; los resultados para las pruebas de laboratorio fueron 0\% (Brucelosis), 86\% (DVB), $43 \%$ (IBR), $5 \%$ (Neospora) y $48.3 \%$ (Leptospirosis); en el caso de Leptospirosis 
los serovares encontrados fueron: L. bratislava (48.3\%), L. pomona (14.4\%), L. icterohaemorrhagiae (20.1\%), L. grippotyphosa (1.2\%), L. canicola (6\%) y L. hardjo (30.5\%). Ryan et al., en 2012 realizaron un estudio poblacional en las hembras bovinas utilizadas como nodrizas en Irlanda, con el fin de determinar por primera vez la seroprevalencia de Leptospirosis, el estudio se realizó en 288 hatos ubicados en todo el país y seleccionados al azar, la prueba serológica empleada fue ELISA (sensibilidad 100\% y especificidad $86.67 \%$ ); el resultado de este estudio indicó una seroprevalencia en el hato e individuo del $82.29 \%$ y $41.75 \%$ de $L$. hardjo respectivamente para este país. Junqueira et al., (2006) realizaron un estudio con el objetivo de evaluar el rendimiento reproductivo del ganado de carne en Brasil infectado naturalmente con IBR, DVB y L. hardjo; los resultados de seroprevalencia fueron $68.3 \%, 98 \%$ y $78.8 \%$ respectivamente, de igual forma una presentación del $18.6 \%$ de abortos. Otros estudios reportan una seroprevalencia de Leptospira borgpetersenii serovar hardjo del $72 \%$ en Inglaterra, 11\% en España y $42 \%$ en EE.UU (Alonso et al., 2001; Wikse et al., 2007). Rivera et al. (2004) reportaron que en la Estación Experimental del Trópico del Centro de Investigaciones (IVITA), en Pucallpa, Perú, el 52.2\% de los animales presentaron anticuerpos contra Leptospira; donde la $L$. hardjo presentó una prevalencia de $35 \%$, seguida por L. canicola y L. icterohaemorrhagiae con $14.9 \%$ de prevalencia; estas fueron las únicas especies de Leptospiras encontradas en este estudio. Grooms, (2006) reportó que en Estados unidos la seroprevalencia de L. hardjo en ganado de leche y doble propósito fue de $35-50 \%$. Estos estudios indican que en el ámbito mundial Leptospira hardjo no es el único agente patógeno asociado a las fallas reproductivas y tampoco es la única serovariedad de Leptospira encontrada en bovinos.

Los bovinos infectados con Leptospira hardjo-bovis presentan infección persistente en los túbulos renales proximales, asociado a la eliminación continua por la orina, y en las infecciones con $L$. hardjo-prajitno se ha reportado que tiene predilección por el tracto genital, considerada como una causa importante de infertilidad en bovinos, en el Reino Unido y Brasil (Givens, 2006). La infertilidad se manifiesta como aumento de los servicios por concepción y prolongados intervalos 
entre partos pero la patogénesis no es clara; se cree que la presencia de Leptospira en el útero y los oviductos de las vacas infectadas interfiere con la implantación del embrión u otros eventos tempranos de la preñez (Grooms, 2006). En un estudio realizado en vacas infectadas experimentalmente con $L$. hardjo tipo hardjo bovis y ovocitos infectados in vitro con el mismo serovar, se encontraron títulos de este serovar en orina, tejido renal, líquido folicular y complejo ovocitocumulus, lo cual indica que las Leptospiras pueden infectar el ovario y posteriormente ser transmitidas al oviducto después de la ovulación (Bielanski y Surujballi, 1996). En toros infectados con hardjo bovis se ha reportado que el microorganismo persiste en las vesículas seminales, así como los riñones (Givens, 2006).

En transferencia de embriones se demostró que es posible obtener embriones transferibles de vacas infectadas con hardjo bovis y de ovocitos expuestos a hardjo bovis in vitro si se usan medios de cultivo y de lavado suplementados con niveles adecuados de antibióticos (penicilina y estreptomicina) para el procesamiento de embriones fecundados in vitro (Bielanski y Surujballi, 1996).

En Colombia la Leptospirosis no es una enfermedad de notificación obligatoria (Ochoa et al., 2000). En Colombia la seroprevalencia para Leptospira en humanos son: L. icterohaemorrhagiae, L. canicola, L. pomona, registrándose una mayor presentación en Atlántico, Bolivar, Tolima, Huila, Quindío; Rodríguez, (2000) reporta que en caninos los serovares más frecuentes son L. canicola, L. pomona; en porcinos: L. pomona, L. bratislava, L. tarassovi; y en bovinos: $L$. hardjo, $L$. pomona, L. canicola, L. grippotyphosa. En la región Caribe se reporta una positividad del $38.2 \%$, en Piedemonte Llanero $24.8 \%$, y Región Andina $14.4 \%$; es así como el promedio para el país se estima en $21.7 \%$. El grado de infección por Leptospira en bovinos en Colombia es muy alto, probablemente alrededor del $50 \%$ (Orrego, 2003). Un estudio realizado por Ochoa et al., (2000) estimó la prevalencia de la infección por Leptospira en operarios, bovinos y porcinos de explotaciones ganaderas de la zona Andina Colombiana, el estudio reportó una prevalencia de $22.4 \%$ en operarios, $60.9 \%$ en las vacas en producción, $10.3 \%$ en los cerdos de 
ceba y $25.7 \%$ en los cerdos de cría ubicados en el departamento de Antioquia (Tabla 8) para este estudio los serotipos más prevalentes fueron bratislava (48.3\%) y hardjo (30.5\%). Bermúdez et al., (2010) realizaron un estudio para establecer la seroprevalencia de Leptospira spp. en la población canina y humana de algunos barrios de Tunja, reportan encontrar una seropositividad del $67.2 \%$ en caninos y $27.1 \%$ en humanos, en los dos grupos se reportaron la presencia de $L$. hardjo con una seroprevalencia de $9.85 \%$ en caninos y $2.17 \%$ en humanos. Estos estudios indican que en Colombia la seroprevalencia para Leptospira en bovinos oscila entre un $50 \%$ y $60.9 \%$, en humanos es en promedio $22 \%$, en caninos el $67.2 \%$ y en cerdos oscila entre $10.3 \%$ y $25.7 \%$ (Ochoa et al., 2000; Rodríguez, 2000; Orrego, 2003; Bermúdez, 2010).

Tabla 8. Porcentaje de individuos positivos a los diferentes serotipos de Leptospira en vacas en producción y en operarios de granjas en Antioquia Serotipos

\begin{tabular}{ccccccc}
\hline Especie & $\begin{array}{c}\text { bratislava } \\
(\%)\end{array}$ & $\begin{array}{c}\text { hardjo } \\
(\%)\end{array}$ & $\begin{array}{c}\text { icterohae- } \\
\text { morrhagiae } \\
(\%)\end{array}$ & $\begin{array}{c}\text { pomona } \\
(\%)\end{array}$ & $\begin{array}{c}\text { canicola } \\
(\%)\end{array}$ & $\begin{array}{c}\text { grippotyphosa } \\
(\%)\end{array}$ \\
\hline Bovinos & $84(48,3)$ & 53 & $35(20.1)$ & $25(14,4)$ & $10(6,0)$ & $2(1,2)$ \\
Operarios & $4(6,0)$ & $3(4,5)$ & $1(1,5)$ & $9(13,5)$ & 0 & 0 \\
\hline
\end{tabular}

Fuente: Ochoa et al., (2000).

En un hato de ganado los factores de riesgo para una Leptospirosis debido a $L$. hardjo incluyen: grandes tamaños del hato, co-pastoreo con ganado infectado $u$ ovejas, acceso del ganado a los cursos de agua contaminados, inadecuadas prácticas de manejo y el reemplazo de animales de vientre. Pero estos factores pueden variar mucho en diferentes partes del mundo, por ejemplo, en EE.UU., la mayor probabilidad de infección con L. borgpetersenii serovar hardjo fue en estados con altas temperaturas medias anuales y largos periodos de cría; en Rio de Janeiro, el principal factor fue el co-pastoreo con otras especies, cerdos principalmente y en Irlanda, el principal factor fue las grandes agrupaciones de ganado (Ryan et al., 2012). En Colombia los serovares de Leptospira han sido $L$. hardjo, L. pomona, L. canicola, L. grippotyphosa (Rodríguez, 2000). 
Los animales que se recuperan de la leptospirosis pueden convertirse en portadores asintomáticos portadores de Leptospiras virulentas en los túbulos renales durante largos períodos y liberar Leptospiras infecciosas en el medio ambiente; otras especies como el ratón (Mus musculus) y ratas (Rattus norvegicus y principalmente Rattus rattus) sirven como reservorios para sus propios serovares (para ratones ballum, icterohaemorrhagiae y para ratas copenhageni); estos animales por lo general, no muestran signos de la enfermedad (Adler y De la Peña, 2010); pero su importancia radica en que las Leptospiras tienen la capacidad de ubicarse en los túbulos renales ya que atraviesa los espacios intertubulares y las células epiteliales de los túbulos, ingresando finalmente en la luz tubular; allí forman microcolonias que se multiplican y finalmente se eliminan por orina (Odriozola, 2001), estas características hacen a las Leptospiras inaccesibles a los anticuerpos, siendo eliminadas en la orina hasta dos o más años, convirtiéndose el animal infectado en la principal fuente de infección para los animales susceptibles. El serovar hardjo puede persistir durante 97 hasta 142 días en el útero de vacas sexualmente maduras (Lopes et al., 2010). La tasa de mortalidad es baja en bovinos (5\%), la morbilidad suele ser elevada, según datos clínicos y serológicos, pudiendo llegar a $100 \%$ de los animales expuestos. En los terneros, la mortalidad es mayor que en los adultos.

Las cifras de abortos (que llegan a un $30 \%$ ), el descenso de la producción de leche y la muerte de bovinos, elevan las pérdidas económicas (Gasque, 2008). Aunque Adler et al., (2011) reportan que L. hardjo nunca causa Leptospirosis fatal, en un estudio a gran escala en Canadá, el serotipo hardjo-bovis fue el más prevalente, donde el serovar hardjo causó alrededor de un $6 \%$ de los abortos, mientras que, en un estudio en EE.UU. se atribuyó como la causa del $10 \%$ de los abortos (Grooms, 2006).

\section{RESPUESTA INMUNE}

La mayoría de las especies animales presentan inmunidad humoral contra la Leptospirosis, esta puede ser conferida a través de la transferencia pasiva de anticuerpos; estos anticuerpos protectores están dirigidos principalmente contra 
los lipopolisacáridos, presentando un problema para el desarrollo de las vacunas con protección cruzada ya que hay más de 220 serotipos diferentes de Leptospira spp. Estos anticuerpos pueden opsonizar células para ser fagocitadas. En los bovinos la respuesta inmune se diferencia de otras especies por la relación entre la respuesta Th 1 mediada por la producción de IFN-y a partir de células $T C_{4}$ gamma/delta (Murray, 2013). La inmunidad adquirida naturalmente ocurre en función de la respuesta humoral mediada, siendo a su vez específica al serovar. El desarrollo de la respuesta humoral se encuentra relacionada por la activación del mecanismo dependiente de los receptores Tool-like tipo 2 (TLR-2), vía sistema inmune innato que es activado por los LPS Leptospíricos. Las Leptospiras pueden activar la proliferación de células $T \gamma-\delta-\alpha-\beta$ (gamma, delta, alfa, beta) (Marinho, 2008).

\section{FISIOPATOLOGÍA DE LA ENFERMEDAD}

Después de penetrar por la piel o por la mucosa, el microorganismo tiene un periodo de incubación de 4 a 10 días, en el cual se multiplica rápidamente y se disemina en ciertos órganos como hígado, riñones, pulmones, tracto reproductor (placenta) y líquido cefalorraquídeo (Gasque, 2008), las lesiones debido a la acción de las toxinas o componentes celulares tóxicos generan la aparición de síntomas consiguientes. La lesión primaria es el daño al endotelio de los vasos sanguíneos pequeños que conducen a isquemia localizada en los órganos, lo que resulta en la necrosis tubular renal, daño hepatocelular y pulmonar, meningitis, miositis y placentitis, las hemorragias se producen en casos graves como ictericia, y con frecuencia, la deficiencia de plaquetas, generalmente hay una granulocitosis leve y esplenomegalia, una vez que aparecen los anticuerpos circulantes, las Leptospiras se eliminan de la circulación y los tejidos por opsonofagocitosis. El daño en los tejidos, a pesar de que es grave, puede ser reversible y seguido por la reparación completa (riñón e hígado), aunque el daño de larga duración (miocarditis) puede ser una complicación y puede conducir a la cicatrización (Adler y de la Peña, 2010). 


\section{Forma aguda}

Durante el periodo temprano de septicemia puede producirse suficiente hemolisina para causar hemoglobinuria, producto de la hemólisis intravascular extensa, esto es frecuente en terneros, no así en animales adultos. Si el animal sobrevive a esta fase, es probable el inicio de un proceso infeccioso en el riñón. El hecho de que se produzca o no hemólisis, depende de que el serotipo particular produzca hemolisina. El daño capilar es común a todos los serotipos y, durante la fase septicémica, las hemorragias petequiales en la mucosa constituyen la expresión de ese daño. Por otra parte, también ocurre daño vascular en el riñón cuando la hemólisis es intensa, se suman a esta lesión vascular básica, anemia, nefrosis y hemoglobinuria. La lesión renal se debe a que la infección persiste en este órgano tiempo después de haber desaparecido en otras localizaciones tisulares. En la fase aguda, el animal puede morir de septicemia, anemia hemolítica o por combinación de ambas. La muerte se deberá a una uremia causada por nefritis intersticial.

\section{Forma subaguda (infección oculta)}

La patogenia es similar a la forma de septicemia aguda, excepto porque la reacción es menos grave. Se observa en todas las especies, pero es más común entre bovinos y equinos adultos.

\section{Forma crónica}

Una secuela frecuente, después de la invasión generalizada, es el aborto provocado por la muerte del feto, con degeneración placentaria o sin ella, en ambos casos, se trata de los efectos resultantes de la invasión al órgano (Figura 4) durante la fase septicémica de la enfermedad. Por lo general, los abortos se presentan de 3 a 10 semanas post-infección (Rodning, 2012), con mayor frecuencia en la segunda mitad de la preñez, pero puede ocurrir en cualquier momento, a partir de los cuatro meses de la gestación (Gasque, 2008). Aunque el aborto ocurre frecuentemente en bovinos y equinos después de la forma aguda 0 
subaguda, también es posible que se produzca sin enfermedad clínica previa (Bermúdez, 2008).

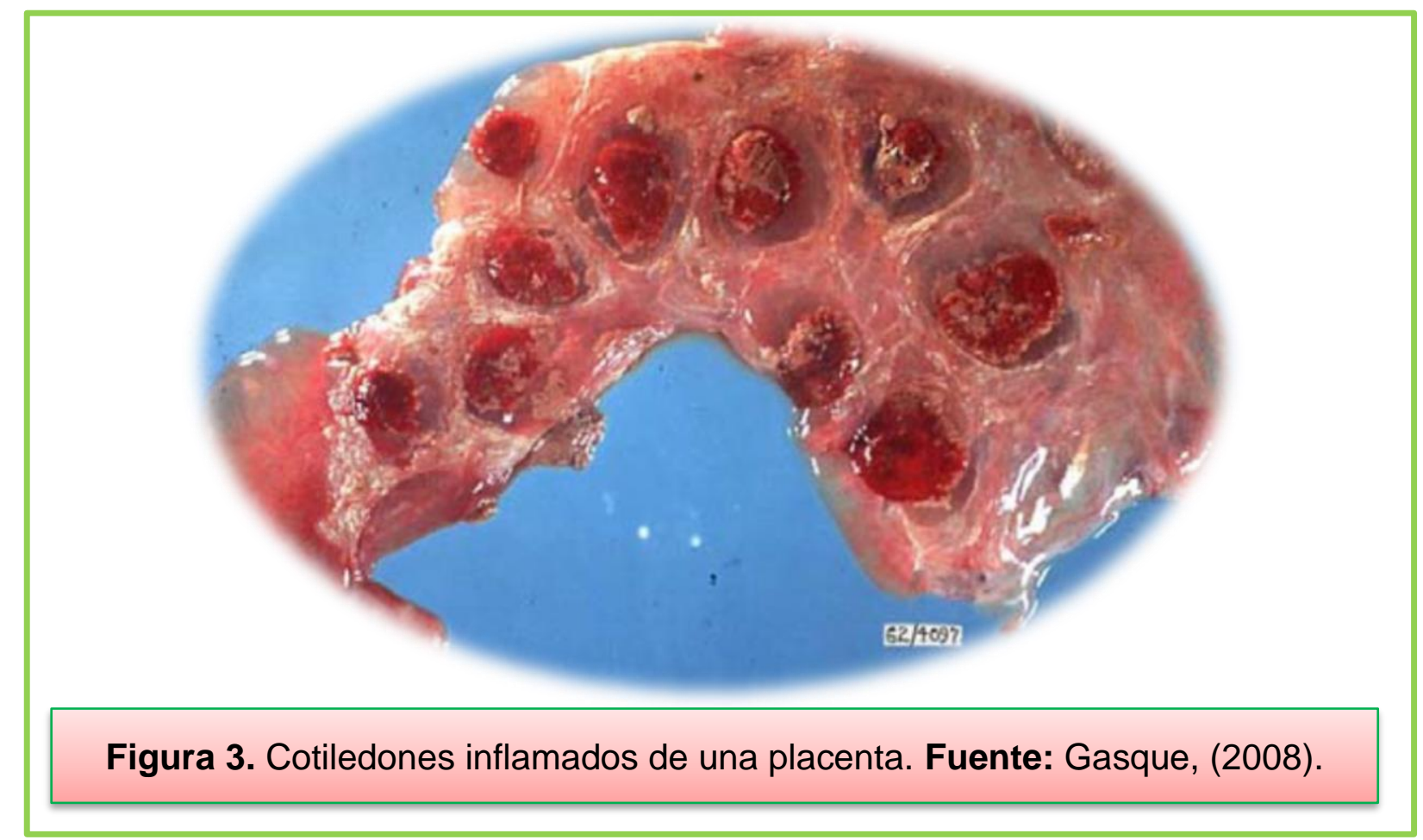

El agente etiológico se puede localizar directamente en la placenta después de una infección sistémica, también se encuentra en la trompa uterina descendiendo al útero y la placenta durante el embarazo, ocasionando placentitis crónica cotiledonaria fibrosante y anemia, causando emaciación fetal (Bermúdez, 2008).

Los cotiledones se observan de color marrón claro y edematosos y las áreas intercotiledonarias amarillentas, el feto muere debido a la placentitis y posterior avascularizacion de los cotiledones (Merck, 2013). Las sustancias toxicas liberadas por los anticuerpos como método de defensa causan lisis de los eritrocitos y presumiblemente atraviesan la barrera placentaria produciendo la muerte fetal por anoxia terminando en aborto (Rodríguez et al., 2005). Este aborto se da 1 a 2 días después de la muerte fetal (Merck, 2013).

\section{CONSIDERACIONES FINALES}

Según los estudios realizados a nivel mundial, se puede decir que Leptospira borgpetersenii serovar hardjo, tipo hardjo bovis y Leptospira interrogans serovar 
hardjo, tipo hardjo prajitmo no son los únicos serovares asociados a la Leptospirosis bovina, pues se ha reportado la presencia de serovares como $L$. icterohaemorrhagiae, L. canicola, L. grippotyphosa, L. bratislava y L. pomona, los cuales pueden presentar síntomas similares a los generados por $L$. hardjo, es de anotar que la Leptospirosis no en la única enfermedad que causa falla reproductiva, pues también se reporta DVB, IBR, neosporosis y brucelosis.

El reporte del hallazgo de los diferentes serovares de Leptospiras en bovinos puede ser debido a que la transmisión del agente patógeno es de forma horizontal directa o indirecta, y a que la multiplicación en los órganos dependa de las condiciones ambientales adecuadas, de esta manera se pueden presentar focos de Leptospirosis si se tiene animales en grandes condiciones de confinamiento, pastoreo con otras especies animales como ovejas, aguas contaminadas, inadecuado control de los vientres de reemplazo al momento de ingresar a la explotación, ubicación en zonas tropicales con altas temperaturas, animales recuperados de la misma enfermedad que permanecen como portadores de la enfermedad.

Finalmente, gran parte del control de la enfermedad depende del profesional a cargo de la explotación, por ello es importante que las personas relacionadas con estas producciones tengan pleno conocimiento, ya que no puede tratarse como un problema individual sino como un problema del hato. También cabe resaltar que a medida que la enfermedad se hace endémica la severidad clínica disminuye gracias a la inmunidad y a los mecanismos de resistencia frente a la patogenicidad del microorganismo y que se puede presentar en asociación con otras enfermedades reproductivas.

Los estudios realizados para determinar las características genotípicas de los serovares de Leptospiras indican que estas especies sufren cambios evolutivos como respuesta al ciclo de transmisión de hospedero a hospedero, esto indica que es necesario el continuo estudio de los serovares para controlar la presencia de una nueva genomoespecie. 
No es correcto clasificar las especies de Leptospiras patógenas y saprófitas en los grupos interrogans y biflexa respectivamente, ya que se encuentran serovares patógenos entre los anteriormente clasificados en la especie biflexa y viceversa. Se puede encontrar un mismo serovar en diferentes especies animales domesticas o silvestres, así como en una sola especie animal se pueden encontrar diferentes serovares de Leptospiras.

Un animal puede estar infectado por serovares de Leptospiras mantenidos por su propia especie o por otras especies, indicando que la transmisión de estos agentes puede ser tanto horizontal como vertical, en la misma especie o en especies diferentes.

Algunos biotipos son similares en la inmunogenicidad, pero no se comportan similarmente en la antigenicidad, lo que los hace indistinguibles en la prueba de microaglutinación (MAT), a pesar de ser molecularmente diferentes. Las Leptospiras inducen inmunidad de tipo humoral, ya que se ha observado que algunos animales siendo hospederos naturales de un serovar en particular no muestran efectos de la enfermedad, pero pueden llegar a desarrollar los síntomas de la enfermedad si son infectados con un serovar distinto, demostrando que la inmunidad solo protege frente al serovar natural, indicando que estos animales inmunes frente a determinado serovar pueden actuar como fuente de infección del mismo.

Los factores de riesgo para una Leptospirosis por L. hardjo incluyen: grandes tamaños del hato, co-pastoreo con ganado infectado u ovejas, acceso del ganado a aguas contaminadas, inadecuadas prácticas de manejo, el reemplazo de animales de vientre, altas temperaturas medias anuales y largas temporadas de cría.

Los animales que se recuperan de la Leptospirosis pueden convertirse en portadores asintomáticos de Leptospiras virulentas en los túbulos renales durante largos períodos y así liberar Leptospiras infecciosas en el medio ambiente. En la fase aguda de la Leptospirosis, el animal puede morir de septicemia, anemia 
hemolítica o por combinación de ambas, la muerte se deberá a una uremia causada por nefritis intersticial. El aborto se produce gracias a la anoxia fetal producida por la placentitis como resultado de las toxinas liberadas por los anticuerpos como método de defensa, los cuales causan lisis de los eritrocitos que posteriormente atraviesan la barrera placentaria, el aborto se presenta de 1 a 2 días después de la muerte fetal.

\section{CONCLUSIONES}

Actualmente la Leptospirosis continúa siendo una enfermedad zoonótica y distribuida mundialmente, reportándose recientemente en Irlanda, serovares en humanos y animales, también se continúan estudios donde se determina la seropositividad a diferentes serovares de Leptospira en humanos y animales silvestres y domésticos en países como Brasil, EE.UU, Ecuador, Inglaterra, España, Perú, Colombia, Suiza, Australia, México entre otros, lo cual refuerza el concepto de enfermedad zoonótica de gran importancia a nivel mundial.

Según las características genotípicas o fenotípicas las Leptospiras pueden estar clasificadas en especies como: L. interrogans y L. biflexa (por sus características fenotípicas), ó en tres genomoespecies: L. interrogans, L. biflexa y L. kirschneri (por sus características genotípicas). Los serovares más prevalentes son $L$. icterohemorragiae, L. pomona, L. grippotyphosa, L. canicola, L. tarssovi, L. hardjo y L. ballum.

En los bovinos la Leptospira borgpetersenii serovar hardjo, tipo hardjo bovis es un serovar adaptado, y la Leptospira interrogans serovar hardjo, tipo hardjo prajitno de la especie interrogans no es el único agente patógeno asociado a las fallas reproductivas y tampoco es la única serovariedad de Leptospira encontrada en bovinos. La Leptospirosis por L. borgpetersenii serovar hardjo tipo hardjo bovis es la causa más reportada en ganado de leche y carne en algunos países del continente americano y se encuentra relacionada pérdidas embrionarias en Brasil, Suiza, Australia, México, EE.UU e Irlanda. 
Los serovares encontrados en Colombia son L. icterohaemorrhagiae, L. canicala, L. pomona, L. bratislava, L. tarassovi, L. grippotyphosa; reportados en humanos, porcinos y bovinos, en las zonas del piedemonte llanero y región andina, con un promedio estimado de $21.7 \%$.

La Leptospira interrogans, serovariedad hardjo, se excreta por el aparato genital bovino durante el aborto e incluso hasta 8 días después del mismo y puede persistir durante 97 a 142 días en el útero de vacas sexualmente maduras. En bovinos la transmisión de $L$. hardjo es más frecuente de forma horizontal directa, mientras que la transmisión indirecta tiene un papel importante en infecciones accidentales, posterior a la exposición del animal a un ambiente contaminado con material infectado por Leptospiras.

En Colombia la seroprevalencia para Leptospira en bovinos oscila entre un 50\% y $60.9 \%$, en humanos es en promedio $22 \%$, en caninos el $67.2 \%$ y en cerdos oscila entre $10.3 \%$ y $25.7 \%$. La forma de transmisión más frecuente del animal al hombre es el contacto de la piel erosionada o mucosas con agua o barro contaminados con orina infectada.

\section{BIBLIOGRAFÍA}

1. Acosta H., Moreno C., Viáfara D. Leptospirosis. Revisión del tema. Colombia Médica, 8 (25): 36-42. 1994.

2. Adler B., de la Peña A. Leptospira and Leptospirosis. Veterinary Microbiology, 140 (34): 287-296. 2010.

3. Adler B., Lo M., Seemann T., Murray G. Pathogenesis of Leptospirosis: The influence of genomics. Veterinary Microbiology, 153 (1-2): 73-81. 2011.

4. Alfaro C., Aranguren Y., Clavijo A. Epidemiologia y diagnóstico de la Leptospirosis como fundamentos para el diseño de estrategias de control. Revista Digital del Centro Nacional de Investigaciones Agropecuarias de Venezuela, Ceniap, Vol. 6. 2004. Disponible http://sian.inia.gob.ve/repositorio/revistas tec/ceniaphoy/articulos/n6/arti/alfaro c/arti/ alfaro c.htm

5. Alonso A., García P., Ortega M. Epidemiologia, diagnóstico y control de la Leptospirosis bovina (Revisión). Investigación Agropecuaria: Producción Sanidad Animal, 16 (2): 205-225. 2001.

6. Araujo M., Suarez V., Gómez J., Céspedes M. Morales A. Leptospirosis. Of. Generalidades de Epidemiología. Instituto Nacional de Salud. Lima, Perú. 2010.

7. Bermúdez V. Importancia del control de Leptospira borgpetesenii serovar hardjo tipo hardjo bovis en el rebaño bovino. En: Desarrollo Sostenible De Ganadería Doble Propósito, p 270-280. 2008. 
8. Bermúdez S., Pulido M., Andrade R. Seroprevalencia de Leptospira spp en caninos y humanos de tres barrios de Tunja, Colombia. Revista MVZ Córdoba, 15 (3): 21852193. 2010.

9. Bielanski A., Surujballi O. Association of Leptospira borgpetesenii serovar hardjo type hardjo bovis with bovine ova and embryos produced by in vitro fertilization. Theriogenology, 46: 45-55. 1996.

10. Cangahuamin R. Diagnóstico de problemas reproductivos en hembras bovinas de la comunidad San Francisco de Toacazo. Sangolquí, Ecuador. Tesis de grado para optar al título de Ingeniero Agropecuario, Escuela Politécnica del Ejercito, 91 p. 2011.

11. Cao X., Dai J., Xu H., Nie S., Chang X., Hu B. Y. et al. High-Coverage proteome analysis reveals the first insight of protein modification systems in the pathogenic spirochete Leptospira Interrogans. Cell Research, 20 (2): 197-210. 2010.

12. Chen L., Xiong Z., Sun L., Yang J., Jin Q. Vfdb Toward the genetic diversity and molecular evolution of bacterial virulence factors. Nucleic Acids Research, 40: 641645. 2012.

13. Cullen P., Haake D., Adler B. Outer membrane proteins of pathogenic spirochetes. Revista Microbiology FEMS, 28 (3): 291-318. 2004.

14. Dey S., Madhan C., Mohan S., Kumar T., Ramadass P., Naimar A., Nachimuthu K. Recombinat LipL32 antigen-based single serum dilution ELISA for detection of canine Leptospirosis. Veterinary Microbiology, 103 (1-2): 99-106. 2004.

15. Dong H., Hu Y., Xue F., Sun D., Ojcius D. M., Mao Y. et al. Characterization of the ompL1 gene of pathogenic Leptospira species in China and cross-immunogenicity of the OmpL1 protein. BMC Microbiology, 8 (223). 2008. Disponible En: http://www.biomedcentral.com/content/pdf/1471-2180-8-223.pdf

16. Ellis W. Leptospirosis como causa de una falla reproductiva. Vet Clin North Am Food Anim Pract., 10: 463-478. 1994.

17. Gasque G. R. Enciclopedia Bovina. Enfermedades de los Bovinos. Leptospirosis. $2^{a}$ Ed. Universidad Nacional Autónoma de México. 168 p. 2008.

18. Givens D. A clinical, evidence based approach to infectious causes of infertility in beef cattle. Theriogenology, 66 (3): 648-654. 2006.

19. Grooms D. L. Reproductive losses caused by bovine viral diarrhea virus and leptospirosis. Theriogenology, 66 (3) 624-628. 2006.

20. Haake D., Suchard M., Kelley M., Dundoo M., Alt D., Zuerner R. Molecular evolution and mosaicism of Leptospiral outer membrane proteins involves horizontal DNA Transfer. Journal of Bacteriology, 186 (9): 2818-2828. 2004.

21. IQB. Diccionario Medico. Recuperado Marzo, 2013. Disponible En: www.lab.Es/Dicaio/E/En

22. Jiménez L. Revisión actualizada sobre métodos de identificación y diagnóstico de leptospirosis en bovinos. Tesis de Grado, Pontificia Universidad Javeriana, Bogotá, 2006.

23. Junqueira R., de Freitas J., Alfieri A., Alfieri A. Avaliação do desempenho reprodutivo de um rebanho bovino de corte. Semina: Ciências Agrárias, 27 (3): 471-480. 2006.

24. Laguna V. Leptospirosis. Módulos Técnicos. Oficina general de Epidemiología, Instituto Nacional de Salud. Serie Documentos Monográficos, N. 2. Lima, 2000.

25. Levett P. Leptospirosis. Clinical Microbiology Reviews, 14 (2) 296-326. 2001.

26. Lopes L., Oliveira A., Melo C., McManus C., Leite R. Efeito da infecção por Leptospira Sp. na eficiência reprodutiva de um rebanho bovino mestiço no sul da Bahia. Revista Brasilera de Medicina Veterinaria, 32 (2): 51-54. 2010.

27. Marinho, M. Leptospirose: Factores epidemiológicos, fisiopatológicos e inmunopatogénicos. Revista Veterinaria e Zootecnia, 15 (3): 428-434. 2008. 
28. Maris S., Rango M. Leptospirosis: Revisión del tema a propósito de dos casos. Revista Biomedicina, Medicina de Emergencia, 6 (2): 38-49. 2011.

29. Merck. The Merck Veterinary Manual. Aborto en ganado. Editorial Staff. 2013.

30. Murray G. The Lipoprotein Lipl32, an enigma of Leptospiral Biology. Veterinary Microbiology, 162 (2-4): 305-314. 2013.

31. Ochoa J. E., Sánchez A., Ruiz I. Epidemiologia de la Leptospirosis en una zona andina de producción pecuaria. Revista Panamericana de Salud Pública, 7 (5): 325331. 2000.

32. Odrizola, E. Leptospirosis. Grupo de Sanidad Animal, Estación Experimental Agropecuaria Balcarce Inta. 2001. Disponible En: http://www.produccionanimal.com.ar/sanidad intoxicaciones metabolicos/enfermedades reproduccion/62leptospirosis.pdf

33. Orrego A. Epidemiologia y diagnóstico de la Leptospirosis bovina. Manejo integrado de plagas y enfermedades en explotaciones ganaderas. Fedegan. 2003.

34. Pérez M. Las más importantes enfermedades genitales de los bovinos (profilaxis, tratamiento, higiene de la recogida del esperma). Rev. Sci. Tech. Off. Int. Epiz., 4 (1): 89-109. 1985.

35. Pedraza A., Salamanca E., Ramírez R., Ospina J., Pulido M. Seroprevalencia de anticuerpos anti-Leptospira en trabajadores de plantas de sacrificio animal en Boyacá, Colombia. Infectio, 16 (1): 30-36. 2012.

36. Rodning S. Leptospirosis in Cattle. Revista Alabama A\&M and Auburn Universities. Revised March. 2012.

37. Rodríguez G. Estado actual de la Leptospirosis. Revista MVZ Córdoba, 5 (1): 61-63. 2000.

38. Rodrigues C., Mortalidade Embrionária / Fetal em Programas de TE (Moet). Revista Brasileira de Reproducao Animal, 27 (2): 64-68. 2003.

39. Rodríguez O., Cuenca J., Tabarez E., Perez R. Leptospira Interrogans. Monografía. 2005. Disponible En: http://www.monografias.com/trabajos25/leptospirainterrogans/leptospira-interrogans.shtml

40. Rodríguez I. El Concepto Serovar en Leptospira (The concept serovar in Leptospira). Revista Electrónica REDVET, 12 (7): Art. 7. 2011.

41. Rivera P., Ticlla M., Balda L., González D., Céspedes M. Diversidad genética de aislamientos peruanos de Leptospira spp. mediante electroforesis en gel de campo pulsado. Revista Peruana de Medicina Experimental y Salud Pública. 29 (4): 469-476. 2012.

42. Ryan E., Leonard N., O`Grady L., Doherty M., More S. Herd-level risk factors associated with leptospira hardjo seroprevalence in beef / suckler herds in the Republic of Ireland. Irish Veterinary Journal, 65: Art. 6. 2012.

43. Ryan E., Leonard N., O'Grady L., More S. J., Doherty M. L. Seroprevalence of Leptospira hardjo in the Irish suckler cattle population. Irish Veterinary Journal, 65: Art. 8. 2012.

44. Wikse S., Rogers G., Ramachandran S., Engelken T., Epperson W., Larson R., Maas J., Richey E., Bolin C. Herd prevalence and risk factors of Leptospira infection in beef cow/calf operations in the United States: Leptospira borgpetersenii serovar hardjo. bovine practitioner, 41 (1): 15-23. 2007.

45. Zuino E., Pizarro R. Leptospirosis: Puesta al día. Revista Chilena de Infectologia, 24 (3): 220-226. 2007. 